International Journal of Pure and Applied Mathematics

Volume 106 No. $4 \quad 2016,977-987$

ISSN: 1311-8080 (printed version); ISSN: 1314-3395 (on-line version)

url: http://www.ijpam.eu

doi: 10.12732/ijpam.v106i4.1

\title{
SOLVING SYSTEM OF HIGHER-ORDER LINEAR DIFFERENTIAL EQUATIONS ON THE LEVEL OF OPERATORS
}

\author{
Srinivasarao Thota ${ }^{1}$, Shiv Datt Kumar ${ }^{2}$ \\ ${ }^{1}$ Department of Mathematics \\ Motilal Nehru National Institute of Technology Allahabad \\ Allahabad, Uttar Pradesh 211004, INDIA
}

\begin{abstract}
In this paper, we present a method for solving the system of higher-order linear differential equations (HLDEs) with inhomogeneous initial conditions on the level of operators. Using proposed method, we compute the matrix Green's operator as well as the vector Green's function of a fully-inhomogeneous initial value problems (IVPs). Examples are discussed to demonstrate the proposed method.
\end{abstract}

AMS Subject Classification: 35E15, 35G10

Key Words: system of higher-order linear differential equations, operator problem, vector Green's function, initial value problems

\section{Introduction}

Many researchers and engineers have vigorously studied the boundary value problems and its applications. Normally, the systems of HLDEs arise in many applications, for example, the models of electrical circuits, multi-body systems, diffusion processes, robotic modelling and mechanical systems, nuclear reactors, dissipative operators, vibrating wires in magnetic fields etc.

Received: April 29, 2015

Published: March 6, 2016 (c) 2016 Academic Publications, Ltd.

url: www.acadpubl.eu 
Suppose $\mathcal{F}=C^{\infty}[a, b]$ and $[a, b] \subset \mathbb{R}$. Consider a system of $n$ linear ordinary differential equations of order $l \geq 1$ of the form

$$
A_{l} \mathrm{D}^{l} u(x)+\cdots+A_{1} \mathrm{D} u(x)+A_{0} u(x)=f(x),
$$

where $\mathrm{D}=\frac{d}{d x}$ is a differential operator, $A_{i} \in \mathcal{F}^{n \times n}$, for $i=0, \ldots, l$, are coefficient matrices, $u(x)=\left(u_{1}(x), \ldots, u_{n}(x)\right)^{T} \in \mathcal{F}^{n}$ is unknown vector to determine, and $f(x)=\left(f_{1}(x), \ldots, f_{n}(x)\right)^{T} \in \mathcal{F}^{n}$ is an $n$-dimensional vector forcing function. If $\operatorname{det}\left(A_{l}\right) \neq 0$, then the system (1) is called of the first kind. In this paper, we consider a system of the first kind with constant coefficients. To obtain an unique solution, we must have a set of initial conditions. The number of initial conditions depends on the number of independent solutions of the homogeneous system (1). In other words, the number of initial conditions and the dimension of null space of matrix differential operator of a given system must coincide. Hence, for a system of the first kind, one needs $n l$ initial conditions. Suppose

$$
B_{1} u(x)=c_{1}, \ldots, B_{l} u(x)=c_{l},
$$

are $l$ initial condition vectors, where $B_{i} \in\left(\mathcal{F}^{n \times n}\right)^{*}$ are initial condition operators and $c_{i}=\left(c_{i 1}, \ldots, c_{i n}\right)^{T} \in \mathbb{R}^{n}$ are initial data, for $i=1, \ldots, l$. Thus we get $n l$ conditions.

In order to find the matrix Green's operator and the vector Green's function of the given IVP (1)-(2), we represent the given system in an operator based notations as $T u=f$ and $B_{1} u=c_{1}, \ldots, B_{l} u=c_{l}$ where $T=$ $A_{l} \mathrm{D}^{l}+\cdots+A_{1} \mathrm{D}+A_{0} \in \mathcal{F}^{n \times n}[\mathrm{D}]$ is a surjective matrix differential operator. We treat the operator notations of a IVP as an inverse operator problem. Using the concept of the classical formulation of variation of parameters and the interpolation technique, we solve the inverse operator problem. We can view the system (1)-(2) as fully-inhomogeneous IVP, (both system and initial conditions are inhomogeneous). For dealing with system (1)-(2) as an operator problem, the parameter $f$ in $(1)$ and $c_{1}, \ldots, c_{l}$ in $(2)$ are necessary. Now the aim is to find the Green's operator $G$ such that $u=G\left(f ; c_{1}, \ldots, c_{l}\right)$ and $B_{1} G\left(f ; c_{1}, \ldots, c_{l}\right)=c_{1}, \ldots, B_{l} G\left(f ; c_{1}, \ldots, c_{l}\right)=c_{l}$, for given $T$ and $B_{1}, \ldots, B_{l}$ with $c_{1}, \ldots, c_{l}$.

Solutions of the differential systems of particular order have been studied by many researchers, for example, in [1, 2, 3], authors presented the resolution of a system of second order differential equations by different methods. Suksern et. al. [4] have given a complete classification of the general case of linear systems of three ordinary differential equations of second-order. Vakulenko 
et. al. [5] considered a large system of quadratic differential equations and presented a reduction algorithm to reduce the large system to smaller systems under some restrictions. Mukesh [6] presented a new technique to solve higher order ordinary differential equations using modified Adomian decomposition method. Using the technique given in [6], we get an approximation to the exact solution of given system. In [7], S. Thota et. al. presented a new method for solving the system of HLDEs, in which they presented a method for finding the general solution of a given system without considering the initial conditions. In this paper, we present a new algorithm for solving IVPs for system (1)-(2) on the level of operators which produces exact solution. Indeed, this paper presents a new algorithm for finding the matrix Green's operator as well as the vector Green's function of a fully-inhomogeneous IVP. It is clear from general observation that, if we can find $\operatorname{Null}(T)$, then we can solve fully inhomogeneous IVP for HLDEs. There exist many forms to express the general solution of the fully inhomogeneous IVP as the composition of solution of semi-homogeneous IVP (homogeneous system with inhomogeneous initial conditions) and solution of semi-inhomogeneous IVP (inhomogeneous system with homogeneous initial conditions). To find an explicit formula in operator settings for the solution corresponding to general linear system (1)-(2), we use the concept of variation of parameters and interpolation technique as discussed in Section 2.

\section{Proposed Method}

Recall the operator problem defined by (1)-(2) as follows

$$
\begin{aligned}
& T u=f, \\
& B u=c .
\end{aligned}
$$

where $T=A_{l} \mathrm{D}^{l}+\cdots+A_{1} \mathrm{D}+A_{0}, f=\left(f_{1}, \ldots, f_{n}\right)^{T}, B=\left(B_{1}, \ldots, B_{l}\right)^{T}$ and $c=\left(c_{1}, \ldots, c_{l}\right)^{T}$.

If $v_{1}, \ldots, v_{n l}$ are independent solutions of the homogeneous system (1) irrespective of the initial conditions, then the matrix $V=\left[v_{1} \cdots v_{n l}\right]$ is called fundamental matrix. Hence $T V=0$. There are many methods in literature to compute such fundamental matrix of a given matrix differential operator $T$. For example, the classical approach in which we convert given system into first order system $[8,9,10,11,12,13]$. Indeed, if $\tilde{u}^{\prime}=M \tilde{u}$ is first order homogeneous system of (1), then the fundamental matrix is obtained from the first $s$ rows of solution $e^{M x}$, where $M$ is the companion matrix. We call the solution of homogeneous first order system $e^{M x}$ as exponential matrix and denote by $\mathcal{E}$. A 
matrix differential operator $T$ (or system of HLDEs) is called regular if it has a regular exponential matrix, and an IVP (3) is called regular if it has a unique solution, otherwise singular. The following proposition presents an algorithm to check the regularity of a given IVP.

Proposition 1. [14] Let $\mathcal{E}$ be the exponential matrix of the given IVP (3). Then there exists unique solution if and only if $\mathcal{E}$ is a regular matrix at $a$, for fixed initial value $a \in \mathbb{R}$.

The matrix $\mathcal{E}$ at $a$ is called the evaluation matrix, denoted by $\mathcal{E}_{a}$.

\subsection{Semi-Inhomogeneous Initial Value Problems}

Consider a semi-inhomogeneous IVP,

$$
\begin{aligned}
& T u=f, \\
& B_{1} u=\cdots=B_{l} u=0,
\end{aligned}
$$

where $T, B_{1}, \ldots, B_{l}$ are as defined before. For given $f$, we find the solution $u \in \mathcal{F}^{n}$ such that $T u=f, B_{i} u=0$. The following theorem presents the solution of IVP (4); and the key step of the finding matrix Green's operator $G$ for system (4) is the classical technique of variation of parameters $[12,13,7,17]$. In operator notations, we find an operator $G$ such that $T G=1$ and $B G=0$.

Theorem 2. Suppose $T=A_{l} D^{l}+\cdots+A_{1} D+A_{0}$ is a matrix differential operator with regular exponential matrix $\mathcal{E}_{a}$ at initial point $a \in \mathbb{R}$. Then, the system (4) has the unique solution

$$
u=G(f)=\left(\begin{array}{c}
\sum_{k=1}^{n l} v_{1, k} I d^{-1} \sum_{p=1}^{n} d_{p}^{k} f_{p} \\
\vdots \\
\sum_{k=1}^{n l} v_{n, k} I d^{-1} \sum_{p=1}^{n} d_{p}^{k} f_{p}
\end{array}\right) \in \mathcal{F}^{n}
$$

and the matrix Green's operator is

$$
G=\left(\begin{array}{ccc}
\sum_{k=1}^{n l} v_{1, k} I d^{-1} d_{1}^{k} & \ldots & \sum_{k=1}^{n l} v_{1, k} I d^{-1} d_{n}^{k} \\
\vdots & \ddots & \vdots \\
\sum_{k=1}^{n l} v_{n, k} I d^{-1} d_{1}^{k} & \ldots & \sum_{k=1}^{n l} v_{n, k} I d^{-1} d_{n}^{k}
\end{array}\right)
$$


where $I$ is the integral operator (i.e., If $=\int_{a}^{x} f d x$ ), $d$ is the determinant of exponential matrix $\mathcal{E}_{a} \in \mathcal{F}^{n l \times n l}$ and $V=\left[v_{1}, \ldots, v_{n l}\right]$ is the fundamental matrix of $T, d_{j}^{i}$ is the determinant of $\mathcal{E}_{j}^{i}$ obtained from $\mathcal{E}_{a}$ by replacing $i$-th column by the $(n l+j-n)$-th unit vector.

Proof. Using the classical approach, we can transform the given IVP (4) into first order system $\tilde{u}^{\prime}=M \tilde{u}+\tilde{f}$ and $\tilde{u}(a)=0$, where $M \in \mathcal{F}^{n l \times n l}$ is the companion matrix and $\tilde{f}=(0, \ldots, 0, f)^{T}$. The first order system can be written as

$$
\begin{aligned}
& \frac{d}{d x}\left(e^{-M x} \tilde{u}\right)=e^{-M x} \tilde{f} \\
& \tilde{u}(a)=0 .
\end{aligned}
$$

Integrating both sides from $a$ to $x$ with respect to $x$, we obtain $e^{-M x} \tilde{u}=$ $\int_{a}^{x} e^{-M \xi} \tilde{f}(\xi) d \xi$. Since $\mathcal{E}=e^{M x}$, the solution of the first order system is $\tilde{u}=$ $\mathcal{E} \int_{a}^{x} \mathcal{E}^{-1} \tilde{f}(\xi) d \xi$. In symbolic notation, we have

$$
\tilde{u}=\mathcal{E} \mathrm{IE}^{-1} \tilde{f}
$$

where $\mathcal{E} I \mathcal{E}^{-1}$ is the Green's operator of the first order system. Now, the solution of the given IVP (4) is obtained by collecting the first $n$ rows of $\tilde{u}$ as in equation (5). The uniqueness of the solution follows from the fact that homogeneous IVP has the trivial solution.

\subsection{Semi-Homogeneous Initial Value Problems}

Consider a semi-homogeneous IVP,

$$
\begin{aligned}
& T u=0, \\
& B_{1} u=c_{1}, \ldots, B_{l} u=c_{l} .
\end{aligned}
$$

For a given initial data $c_{1}, \ldots, c_{l}$ at initial point $a$, we find $u \in \mathcal{F}^{n}$ such that $T u=0$ and $B_{i} u=c_{i}$. The key step to find matrix Green's operator $G$ for system (6) is the interpolation technique [16] satisfying the initial conditions. In operator notations, we find $G$ such that $T G=0$ and $B G=C$, where $C=\left(\operatorname{diag}\left(c_{1}\right), \ldots, \operatorname{diag}\left(c_{l}\right)\right)^{T}$, as in the following theorem. 
Theorem 3. Suppose $T$ is a matrix differential with a regular exponential matrix $\mathcal{E}_{a}$ at initial point $a \in \mathbb{R}$. Then, the system (6) has the unique solution

$$
u=G\left(c_{1}, \ldots, c_{l}\right)=\left(\begin{array}{c}
\sum_{k=1}^{n l} v_{1, k} E d^{-1} \sum_{p=1}^{n l} d_{p}^{k} \tilde{c}_{p} \\
\vdots \\
\sum_{k=1}^{n l} v_{n, k} E d^{-1} \sum_{p=1}^{n l} d_{p}^{k} \tilde{c}_{p}
\end{array}\right) \in \mathcal{F}^{n}
$$

where $E$ is the evaluation operator (i.e., $E f(x)=f(a)$ ), $d$ is the determinant of exponential matrix $\mathcal{E}_{a} \in \mathcal{F}^{n l \times n l}$ and $V=\left[v_{1}, \ldots, v_{n l}\right]$ is fundamental matrix of $T$; $d_{j}^{i}$ is the determinant of $\mathcal{E}_{j}^{i}$ obtained from $\mathcal{E}_{a}$ by replacing $i$-th column by the $j$-th unit vector; and $\left(\tilde{c}_{1}, \ldots, \tilde{c}_{n l}\right)^{T}=\left(c_{1}, \ldots, c_{l}\right)^{T}=c$. The matrix Green's operator is

$$
G=V E \mathcal{E}_{a}^{-1} C
$$

Proof. We have

$$
\begin{aligned}
T u & =\left(\sum_{r=1}^{n} T_{1}^{r} \sum_{k=1}^{n l} v_{r, k} \mathrm{E} d^{-1} \sum_{p=1}^{n l} d_{p}^{k} \tilde{c}_{p}, \ldots, \sum_{r=1}^{n} T_{n}^{r} \sum_{k=1}^{n l} v_{r, k} \mathrm{E} d^{-1} \sum_{p=1}^{n l} d_{p}^{k} \tilde{c}_{p}{ }^{T}\right. \\
& =\left(\sum_{r=1}^{n} \sum_{k=1}^{n l} T_{1}^{r} v_{r, k} \mathrm{E} d^{-1} \sum_{p=1}^{n l} d_{p}^{k} \tilde{c}_{p}, \ldots, \sum_{r=1}^{n} \sum_{k=1}^{n l} T_{n}^{r} v_{r, k} \mathrm{E} d^{-1} \sum_{p=1}^{n l} d_{p}^{k} \tilde{c}_{p}\right)^{T} \\
& =(0, \ldots, 0)^{T}=0
\end{aligned}
$$

for $T V=0$, where $T_{i}^{j}$ is the $j$-th column and $i$-th row of $T$; and $T G=$ $T V \mathrm{E}^{-1} C=0$. Also

$$
\begin{aligned}
B_{i} u & =\left(\mathrm{ED}^{i-1} \sum_{k=1}^{n l} v_{r, k} \mathrm{E} d^{-1} \sum_{p=1}^{n l} d_{p}^{k} \tilde{c}_{p}, \ldots, \mathrm{ED}^{i-1} \sum_{k=1}^{n l} v_{r, k} \mathrm{E} d^{-1} \sum_{p=1}^{n l} d_{p}^{k} \tilde{c}_{p}{ }^{T}\right. \\
& =\left(\sum_{k=1}^{n l} \mathrm{ED}^{i-1} v_{r, k} \mathrm{E} d^{-1} \sum_{p=1}^{n l} d_{p}^{k} \tilde{c}_{p}, \ldots, \sum_{k=1}^{n l} \mathrm{ED}^{i-1} v_{r, k} \mathrm{E} d^{-1} \sum_{p=1}^{n l} d_{p}^{k} \tilde{c}_{p}\right)^{T} \\
& =\left(\tilde{c}_{(i-1) l+1}, \ldots, \tilde{c}_{(i-1) l+l}\right)^{T}=\left(c_{i, 1}, \ldots, c_{i, l}\right)^{T}=c_{i},
\end{aligned}
$$

and hence $B G=C$. The uniqueness of solution follows from the fact that the evaluation matrix $\mathcal{E}_{a}$ is regular. 


\subsection{Fully-Inhomogeneous Initial Value Problems}

The solution of a fully-inhomogeneous system (3) is obtained by composing two solutions of the semi-inhomogeneous IVP (given in Theorem 2) and the semi-homogeneous IVP (given in Theorem 3). The Generalization of this fact is given in the following theorem.

Theorem 4. Suppose $T=A_{l} D^{l}+\cdots+A_{1} D+A_{0}$ is a matrix differential operator with regular exponential matrix $\mathcal{E}_{a}$ at initial point $a \in \mathbb{R}$ and the initial data $c_{1}, \ldots, c_{l}$. Then, the IVP

$$
\begin{aligned}
& T u=f, \\
& B u=c
\end{aligned}
$$

has the unique solution

$$
u=G\left(f ; c_{1}, \ldots, c_{l}\right)=G_{1}(f)+G_{2}\left(c_{1}, \ldots, c_{t}\right) \in \mathcal{F}^{n}
$$

and the matrix Green's operator is

$$
G=G_{1}+G_{2}
$$

where $G_{1}$ and $G_{2}$ are the matrix Green's operators as in Theorem 2 and Theorem 3 respectively.

\section{Examples}

Example 1. Consider a system of equations

$$
\begin{aligned}
& u_{1}^{\prime \prime}=f_{1}, \\
& u_{2}^{\prime \prime}+2 u_{2}^{\prime}+4 u_{1}=f_{2}, \\
& u_{1}(0)=\alpha_{1}, u_{2}(0)=\alpha_{2}, u_{1}^{\prime}(0)=\alpha_{3}, u_{2}^{\prime}(0)=\alpha_{4} .
\end{aligned}
$$

In operator notations, we have

$$
\begin{aligned}
& T=\left(\begin{array}{cc}
\mathrm{D}^{2} & 0 \\
4 & 2 \mathrm{D}+\mathrm{D}^{2}
\end{array}\right) ; u=\left(\begin{array}{l}
u_{1} \\
u_{2}
\end{array}\right) ; f=\left(\begin{array}{l}
f_{1} \\
f_{2}
\end{array}\right) ; \\
& B=\left(\begin{array}{cc}
\mathrm{E} & 0 \\
0 & \mathrm{E} \\
\mathrm{ED} & 0 \\
0 & \mathrm{ED}
\end{array}\right) ; C=\left(\begin{array}{cc}
\alpha_{1} & 0 \\
0 & \alpha_{2} \\
\alpha_{3} & 0 \\
0 & \alpha_{4}
\end{array}\right) ; c=\left(\begin{array}{l}
\alpha_{1} \\
\alpha_{2} \\
\alpha_{3} \\
\alpha_{4}
\end{array}\right) .
\end{aligned}
$$


The system of equations and initial conditions given in (7) can be rewrite as $T u=f$ and $B u=c$. Following the algorithm in Section 2, we have the matrix Green's operator as

$$
G=\left(\begin{array}{ll}
g_{11} & g_{12} \\
g_{21} & g_{22}
\end{array}\right)
$$

where

$$
\begin{aligned}
g_{11}= & x \mathrm{I}-\mathrm{I} x+\mathrm{E}+x \mathrm{ED} \\
g_{12}= & 0 \\
g_{21}= & (2 x-1) \mathrm{I} x+\left(x-x^{2}-\frac{1}{2}\right) \mathrm{I}+\frac{1}{2} e^{-2 x} \mathrm{I} e^{2 x} \\
& -\mathrm{I} x^{2}+\left(-e^{-2 x}-2 x+1\right) \mathrm{E}+\left(\frac{1}{2} e^{-2 x}+x-x^{2}-\frac{1}{2}\right) \mathrm{ED}, \\
g_{22}= & \frac{1}{2} \mathrm{I}-\frac{1}{2} e^{-2 x} \mathrm{I} e^{2 x}+\mathrm{E}+\left(-\frac{1}{2} e^{-2 x}+\frac{1}{2}\right) \mathrm{ED},
\end{aligned}
$$

and the vector Green's function is

$$
u=G\left(f ; \alpha_{1}, \alpha_{2}, \alpha_{3}, \alpha_{4}\right)=\left(\begin{array}{l}
u_{1} \\
u_{2}
\end{array}\right)
$$

where

$$
\begin{aligned}
u_{1}= & x \int_{0}^{x} f_{1} d x-\int_{0}^{x} x f_{1} d x+\alpha_{1}+x \alpha_{3} \\
u_{2}= & (2 x-1) \int_{0}^{x} x f_{1} d x+\left(x-x^{2}-\frac{1}{2}\right) \int_{0}^{x} f_{1} d x \\
& +\frac{1}{2} e^{-2 x} \int_{0}^{x} e^{2 x} f_{1} d x-\int_{0}^{x} x^{2} f_{1} d x+\left(-e^{-2 x}-2 x+1\right) \alpha_{1} \\
& +\left(\frac{1}{2} e^{-2 x}+x-x^{2}-\frac{1}{2}\right) \alpha_{3}+\frac{1}{2} \int_{0}^{x} f_{2} d x \\
& -\frac{1}{2} e^{-2 x} \int_{0}^{x} e^{2 x} f_{2} d x+\alpha_{2}+\left(-\frac{1}{2} e^{-2 x}+\frac{1}{2}\right) \alpha_{4} .
\end{aligned}
$$

If we choose $f=\left(\cos x, e^{x}\right)^{T}$ and $c=(1,0,2,-1)^{T}$ for simplicity, then the exact solution is

$$
u=\left(\begin{array}{c}
2-\cos x+2 x \\
-2 x-\frac{4}{5} \cos x+\frac{8}{5} \sin x+\frac{7}{15} e^{-2 x}+\frac{1}{3} e^{x}-2 x^{2}
\end{array}\right) .
$$


It is clear that $T u=f, B u=C$ and $T G=I, B G=0$.

Example 2. [18] Consider an electrical circuit with a resistance of 6 ohms, an inductance of 0.5 henry, a generator providing alternating voltage given by $24 \sin (10 t)$ for $t \geq 0$ and a capacitance of 0.02 farad. The alternating voltage is the external force applied to the circuit, and the resistance is a damping coefficient. By Kirchhoff's Law, we have following system of equations, if $Q$ is the instantaneous charge on the capacitor,

$$
\begin{aligned}
& u_{1}^{\prime}=u_{2} \\
& u_{2}^{\prime}=48 \sin 10 t-12 u_{2}-100 u_{1},
\end{aligned}
$$

where $u_{1}=Q$ and $u_{2}=\frac{d Q}{d t}$. Let $u_{1}(0)=\alpha_{1}$ and $u_{2}(0)=\alpha_{2}$ be initial conditions. In operator notations, we have

$$
\begin{aligned}
A_{1} & =\left(\begin{array}{ll}
1 & 0 \\
0 & 1
\end{array}\right), A_{0}=\left(\begin{array}{cc}
0 & -1 \\
100 & 12
\end{array}\right) \text { and } T=\left(\begin{array}{cc}
\mathrm{D} & -1 \\
100 & 12+\mathrm{D}
\end{array}\right) \\
B_{1} & =\left(\begin{array}{l}
\mathrm{E} \\
0
\end{array}\right), B_{2}=\left(\begin{array}{l}
0 \\
\mathrm{E}
\end{array}\right) \text { and } B=\left[B_{1}, B_{2}\right]=\left(\begin{array}{ll}
\mathrm{E} & 0 \\
0 & \mathrm{E}
\end{array}\right) ; \\
c & =\left(\begin{array}{l}
\alpha_{1} \\
\alpha_{2}
\end{array}\right) \text { and } C=\left(\begin{array}{cc}
\alpha_{1} & 0 \\
0 & \alpha_{2}
\end{array}\right), f=\left(\begin{array}{c}
0 \\
48 \sin 10 t
\end{array}\right)
\end{aligned}
$$

The exact solution of the given system is computed similar to Example 1 as

$$
u=\left(\begin{array}{l}
u_{1} \\
u_{2}
\end{array}\right),
$$

where

$$
\begin{aligned}
u_{1}= & e^{-6 t}\left(\frac{2}{5} \cos 8 t+\frac{3}{10} \sin 8 t\right)-\frac{2}{5} \cos 10 t \\
& +e^{-6 t} \alpha_{1}\left(\frac{3}{4} \sin 8 t+\cos 8 t\right)+\frac{1}{8} e^{-6 t} \sin 8 t \alpha_{2} \\
u_{2}= & 4 \sin 10 t-5 e^{-6 t} \sin 8 t-\frac{25}{2} e^{-6 t} \sin 8 t \alpha_{1} \\
& -\frac{3}{4} e^{-6 t} \sin 8 t \alpha_{2}+\alpha_{2} e^{-6 t} \cos 8 t
\end{aligned}
$$

Remark: The Example 2 shows the simplicity of the proposed method. In this example, we find the exact solution of IVP with arbitrary boundary data 
unlike the authors presented in [18, p.173] for particular boundary data. i.e. authors solved Example 2 with fixed $\alpha_{1}=\alpha_{2}=0$ and the solution is

$$
u=\left(\begin{array}{c}
e^{-6 t}\left(\frac{2}{5} \cos 8 t+\frac{3}{10} \sin 8 t\right)-\frac{2}{5} \cos 10 t \\
4 \sin 10 t-5 e^{-6 t} \sin 8 t
\end{array}\right) .
$$

We can check easily that $T u=f$ and $B u=c$.

\section{References}

[1] D. Sengupta: Resolution of the identity of the operator associated with a system of second order differential equations, J. of Math. and Comp. Sci., 5, No. 1 (2015), 56-71.

[2] D. Sengupta: On the expansion problem of a function associated with a system of second order differential equations, J. of Math. and Comp. Sci., 3, No. 6 (2013), 1565-158.

[3] D. Sengupta: Asymptotic expressions for the eigenvalues and eigenvectors of a system of second order differential equations with a turning point (Extension II), Int. J. of Pure and App. Math., 78, No. 1 (2012), 85-95.

[4] S. Suksern, S. Moyo, S. V. Meleshko: Application of group analysis to classification of systems of three second-order ordinary differential equations, John Wiley \& Sons, Ltd. (2015).

[5] S. Vakulenko, D. Grigoriev, A. Weber: Reduction methods and chaos for quadratic systems of differential equations. Studies in Applied Mathematics (2015).

[6] M. Grover: A New Technique to Solve Higher Order Ordinary Differential equations. IJCA Proceedings on National Workshop-Cum-Conference on Recent Trends in Mathematics and Computing 2011 RTMC (2012).

[7] S. Thota, S. D. Kumar: A new method for general solution of system of higher-order linear differential equations. International Conference on Inter Disciplinary Research in Engineering and Technology, 1 (2015), 240-243.

[8] R. B. Taher, M. Rachidi: Linear matrix differential equations of higher-order and applications. Elec. J. of Diff. Equ., 95 (2008), 1-12.

[9] G. I. Kalogeropoulos, A. D. Karageorgos, A. A. Pantelous: Higher-order linear matrix descriptor differential equations of Apostol-Kolodner type. Elec. J. of Diff. Equ., 25 (2009), 1-13.

[10] S. Abramov: EG - eliminations. J. of Diff. Equ. and Appl., 5 (1999), 393-433.

[11] C. E. BACHA:Méthodes Algébriques pour la Résolution d'Équations Différentielles Matricicelles d'Ordre Arbitraire. PhD thesis, LMC-IMAG (2011).

[12] E. A. Coddington, N. Levinson: Theory of ordinary differential equations. McGraw-Hill Book Company, Inc. (1955).

[13] E. A. Coddington and R. Carlson: Linear ordinary differential equations. Society for Industrial and Applied Mathematics (SIAM), (1997).

[14] K. Hoffman, R. Kunze: Linear Algebra, second edition. Pearson Education, Inc., India (2008). 
[15] T. Sauer: Polynomial interpolation, ideals and approximation order of refinable functions, Proceedings American Mathematical Society (1997).

[16] S. Thota and S. D. Kumar: Symbolic method for polynomial interpolation with Stieltjes conditions, Proceedings of International Conference on Frontiers in Mathematics (2015), $225-228$.

[17] T. M. Apostol: Calculus, II . John Wiley \& Sons (Asia) Pte. Ltd. (2002).

[18] H. J. Ricardo: A Modern Introduction to Differential Equations, Second Edition, Academic Press, March, (2009). 
